\title{
Monitoring at TeV Energies with M@TE
}

\section{Ruben Alfaro $^{a *}$, Abel Bernal $^{b}$, Thomas Bretz ${ }^{c}$, Simone Dichiara ${ }^{b}$, Daniela Dorner ${ }^{d}$, Fernando Garfias $^{b}$, María Magdalena González ${ }^{b}$, David Hiriart $^{b}$, Arturo Iriarte $^{b}$, Elena Jimenez ${ }^{b}$, Luis Artemio Martínez ${ }^{b}$, Lukas Nellen ${ }^{e}$, Ibrahim Torres ${ }^{f}$, Gagik Tovmasian $^{b}$,}

${ }^{a}$ Instituto de Física, Universidad Nacional Autónoma de México, México D.F., México

${ }^{b}$ Instituto de Astronomía, Universidad Nacional Autónoma de México, México D.F., México

${ }^{c}$ RWTH Aachen University, Physics Institute III A, Aachen, Germany

${ }^{d}$ Universität Würzburg, Institute for Theoretical Physics and Astrophysics, Würzburg, Germany

e Instituto de Ciencias Nucleares, Universidad Nacional Autónoma de México, México D.F., México

${ }^{f}$ Instituto Nacional de Astrofísica, Óptica y Electrónica, Puebla, México

E-mail: rubenefisica.unam.mx

Blazars are extremely variable objects emitting radiation across the electromagnetic spectrum and showing variability on time scales from minutes to years. Simultaneous multi-wavelength observations are crucial for understanding the emission mechanisms. In particular the study of their $\mathrm{TeV}$ emission is relevant to test the dominant radiative process at such energies (e.g. leptonic models predict a correlation between $\mathrm{X}$-ray and $\mathrm{TeV}$ emission). As well, the correlation with the bump at low energy or the possible common detection with a neutrino signal can be relevant to constrain the physical model. From radio via optical and from X-ray to gamma rays, a variety of instruments, as OVRO and Fermi, are already monitoring blazars. At TeV energies, long-term monitoring is currently carried out by HAWC and FACT. Towards 24/7 continuous observations, the goal is to have similar monitoring telescopes at locations around the globe in order to close temporal gaps and compile light curves with homogeneous sensitivity. With the M@TE (Monitoring at $\mathrm{TeV}$ energies) project, we are planning to install an Imaging Air Cherenkov Telescope equipped with an improved version of the FACT camera and the mechanical structure of one of the mounts of the HEGRA experiment at the site of San Pedro Mártir in Mexico. Featuring excellent observation conditions, this location provides a variety of instruments from radio to optical wavelength allowing for coordinated multi-wavelength blazar studies. In this work, we will present the status of the project.

35th International Cosmic Ray Conference - ICRC2017

10-20 July, 2017

Bexco, Busan, Korea

${ }^{*}$ Speaker. 


\section{INTRODUCTION}

Active galactic nuclei (AGN) emit radiation across the whole electromagnetic spectrum. Their spectral energy distributions (SEDs) feature two peaks. While the low energy peak is synchrotron radiation from accelerated particles, the origin of the high energy peak is still under debate. Part of the models consider inverse Compton scattering of the electron population with photons emitted before by the same electrons (Synchrotron Self Compton, SSC) or external photons (External Compton, EC), called leptonic models. Other models consider hadronic acceleration processes.

For the class of blazars, i.e. AGN having their jet pointing towards the observer, the high energy peak is in the $\mathrm{TeV}$ energy range making them an interesting targets in $\mathrm{TeV}$ astronomy.

Another distinct feature is their extreme variability on time scales from minutes to years $[1,2]$. To study the variability and draw conclusions on the emission region and mechanism, time-resolved SEDs are needed.

Long-term monitoring contributes to understand the characteristics of blazars in different ways, e.g.: to follow the source during the different phases of the emission (flaring or quiescent state); to study the spectral evolution and get insight on the physical model; to assess the variability characteristics of blazars on all time-scales from minutes to years.

\subsection{Status of Monitoring at TeV Energies}

As the current generation of large Imaging Air Cherenkov Telescopes (IACTs) cover a wide scope of scientific questions, monitoring has a lower priority. Regular multi-wavelength campaigns have been carried out since 2009 [3, 4, 5], but the monitoring data are still rather sparse [7].

The First G-APD Cherenkov Telescope (FACT, [6]) is an IACT monitoring bright blazars at $\mathrm{TeV}$ energies since more than five years [8]. Thanks to the usage of semiconductor-based photosensors (SiPMs, a.k.a. Geiger-mode avalanche photodiodes), the telescope is ideally suited for long-term monitoring. The stable detector performance provided by the photosensors facilitates the analysis of a long-term data sample [9]. Furthermore, it helps to automate the operation of the instrument [10] which increases the data taking efficiency. The fact that SiPMs do not show any degradation when exposed to bright ambient light, allows for continuous observations minimizing the observational gaps around full moon.

Providing a stable detector performance and optimizing the duty cycle of the instruments, SiPMs are ideal for long-term monitoring. Over the last more than five years, almost 9000 hours of physics data have been recorded with about 2000 hours of Mrk 421 and Mrk 501 each [8]. Apart from that, the Crab Nebula and few other blazars like 1ES 1959+650 and 1ES 2344+51.4 are being observed. Thanks to the automatic operation, a data taking efficiency of more than $90 \%$ has been achieved [13].

The High Altitude Water Cherenkov (HAWC) Observatory is located at 4,100 m a.s.l. at the Parque Nacional Pico de Orizaba in the state of Puebla, Mexico. Since March 2015, it has been operating in its complete configuration. The detector is using the water Cherenkov technique and consists of 300 water tanks instrumenting an area of $22,000 \mathrm{~m}^{2}$. The array is sensitive to extensive air showers induced by gamma rays with energies between approximately $500 \mathrm{GeV}$ and $100 \mathrm{TeV}$ with a peak sensitivity in the range between 2 and $10 \mathrm{TeV}$, depending on source declinations and spectra. Compared to the air Cherenkov technique, it has the advantage of a wider field of view 
( $\sim 2$ steradians) and being independent of weather and sun allowing for almost continuous daily monitoring. HAWC can observe any source that transits through a $45^{\circ}$ cone centered on local zenith for up to 6 hours per day [14]. From a bit more than one year of data [15], mainly Mrk 421 and Mrk 501 are detected by HAWC in the extragalactic sky.

While HAWC provides continuous monitoring, FACT has a better sensitivity [11, 12]. From one site, a maximum of six to eight hours of data can be taken during one night, leaving gaps of 16 to 18 hours. To overcome this, the ultimate goal is a network of Cherenkov telescopes around the globe [16].

\section{M@TE - Monitoring at TeV Energies}

Monitoring at TeV Energies (M@TE) is a bi-national project of German and Mexican institutions. One of its goals is to refurbish an Imaging Air Cherenkov Telescope (IACT) of the former High-Energy Gamma-ray Array (HEGRA) for long-term monitoring. At a distance of about $6 \mathrm{~h}$ to the Canary Islands, Mexico is the ideal complement of the FACT site and significantly prolongs the uninterrupted observation-time accessible per night. Since both telescopes are based on former HEGRA telescopes, M@TE can essentially utilize the same technology as successfully operated in FACT. In Mexico, there are two telescope mounts available, one of them has been already installed at the HAWC site. Due to the old electronics of the HEGRA camera and lack of documentation its operation has been very limited. Therefore the plan is to upgrade all the electronics systems and to replace the round mirrors by hexagonal mirrors which will improve the light collection. In order to reduce time and man power the goal is to stay with a design close to the FACT design. Since new generations of SiPMs and read-out electronics have been developed for CTA, this new technology will be considered in the camera design.

\subsection{Project, Plan and Status}

Infrastructure The installation of one former HEGRA telescope mount at the HAWC site [17] and thus the easy availability of equipment and support from the HAWC observatory, allows to test equipment and procedures at this site. The HAWC site itself is also suitable for the operation of the telescope, but located at 4,100 m, it provides less stable weather conditions than Observatorio Astronomico Nacional (OAN) at San Pedro Mártir. Therefore the second telescope mount will be refurbished and installed there.

Camera The camera design will be similar to the FACT camera [6]. However, the light sensors chosen are MicroJC-60035 from SensL [18]. Those sensors type are ideally suited due to their high photon detection efficiency (PDE) at blue wavelength. The new MicroJC series available since September 2015 increases the photon detection efficiency even further, especially for wavelengths below $350 \mathrm{~nm}[19,18]$. Measurements of these sensors were made at the Physics Institute 3A and 3B at RWTH Aachen University for other projects. The MicroJC-60035 series increases the effective light collection efficiency by $\sim 55 \%$ as compared to the Hamamatsu S10362 used in the FACT camera. The currently available Hamamatsu S13360 devices [20] show an increase of $\sim 33 \%$. The main disadvantage of the SensL MicroJC series is their two times longer recovery time, but this effect can be suppressed by pulse-shaping in the electronics. About the light guides, after 
an optimization process using the ZEMAX software no big improvement was observed modifying the FACT light guides. Therefore the light guides in M@TE will be similar to those FACT. Experiences with the production of the FACT cones showed that for injection molding, better results are obtained if the Plexiglas is injected from the light outlet rather than the side. To reduce costs, a new mechanical-machining procedure is under evaluation.

Mirrors Hexagonal shape mirrors were purchased from the company GALACTICA. They are made of $2.5 \mathrm{~cm}$ thick glass, with $600 \mathrm{~mm}$ size inner-diameter and a curvature of $12,000 \mathrm{~mm}$. Two different focal lengths were chosen in order to account for the difference in distance from the structure mounting point to the camera center. This brings the reflector design closer to a parabolic mirror, thus decreasing the time-spread of an arriving wavefront. Three round plates attached with ball joints will be glued to the mirrors to allow for the alignment of the mirror facets.

Telescope Control Software The FACT collaboration developed a control software with a modular approach that allows the easy application [21]. Therefore, we stay with similar components based on components from Bosch-Rexroth such as the IndraDrive drive controller and the PLC L20. Only minor changes are requested wherever hardware interfaces change. Web-interfaces for scheduling and remote control also will need minor or no adaption. The main goal is to have a robotic system that requires the minimum (or non) human assistance to take data.

Cross-Calibration with HAWC Installing the camera in the telescope at the HAWC site, offers the unique opportunity to cross-calibrate two different measurement techniques at $\mathrm{TeV}$ energies. From this, a lot of information for future multi-messenger high energy observatories can be gained. Furthermore, by comparing the data, the analysis of both instruments can be improved.

Site for Monitoring The HAWC site offers several advantages and many tests will be carry out in Sierra Negra however the high altitude has not been evaluated in terms of performance of IACTs.Besides, the atmospheric conditions in Volcán Sierra Negra are not ideal for this technique. Fherefore the final site of M@TE will be located close to the Observatorio Astronómico Nacional de San Pedro Mártir (OAN-SPM) at 2,830 m a.s.l. which is comparable to the altitude of FACT at $2,200 \mathrm{~m}$ a.s.l. Being located at $30^{\circ} 54^{\prime} 19^{\prime \prime} \mathrm{N}$ and $115^{\circ} 30^{\prime} 04^{\prime \prime} \mathrm{W}$, as compared to the FACT telescope at $28^{\circ} 45^{\prime} 43^{\prime \prime} \mathrm{N}$ and $17^{\circ} 53^{\prime} 24^{\prime \prime} \mathrm{W}$, OAN-SPM is in excellent complement from its geographic location. The chosen site is ideally located for continuous monitoring, given that target objects culminate at nearly identical zenith distances and the sites are offset by about $6.5 \mathrm{~h}$ which matches very well the typical visibility time per night. Furthermore, the site has excellent weather conditions with $\sim 80 \%$ of cloudless and dark skies. It was the runner up site for the CTA North [23]. Since this area is under the supervision of OAN-SPM, no delay or problems are expected to install M@TE.

Status Partial financial support for mobility allows us to start working on the project. Most of the telescope control system are in hand, so late this summer the test in Sierra Negra will begin. The mirrors arrived to Ensenada, Baja California, where their main parameters (Focal length, PSF, reflectivity, etc.) will be re-measured and where the metallic round plate will be glued to attach the mirrors to the mechanical structure. The light sensors and light guides are going to be characterized before gluing them together. The final arrangement or grouping of them will depend on the final 
front-end electronics and data acquisition system. A possible front-end electronics is tested now in a fluorescence telescopes under development at RWTH Aachen University [22].

\section{Summary and Outlook}

Monitoring at TeV Energies (M@TE) is a project installing a FACT-like IACT in Mexico. Partial financial support allow us to start working on mechanical and optical elements. The site has been selected. The telescope will be installed to the Observatorio Astronomico de San Pedro Mártir in the state of Baja California, Mexico and the instrumentation will be tested at the HAWC site where a cross-calibration between water Cherenkov and imaging air Cherenkov technique is possible for the first time. The site in San Pedro Mártir is better suited for long-term monitoring. Like that, continuous and unbiased observations can be extended to up to twelve hours allowing for more detailed studies of blazar variability

\section{Acknowledgements}

We acknowledge the support from Consejo Nacional de Ciencia y Tecnología (CONACYT), México (grant 279446) and DGAPA-UNAM, México (grants IN109916 and IG100317) and the travel funding from German Academic Exchange Service (DAAD) PPP Mexico.

\section{References}

[1] F. Aharonian, A. G. Akhperjanian, A. R. Bazer-Bachi et al., An Exceptional Very High Energy Gamma-Ray Flare of PKS 2155-304, ApJ 664 (2007) L71-L74

[2] J. Albert, J. , E. Aliu, H. Anderhub et al., Variable Very High Energy $\gamma$-Ray Emission from Markarian 501, ApJ 669 (2007) 862-883

[3] S. Aleksić , L. A. Ansoldi, P. Antonelli et al., Unprecedented study of the broadband emission of Mrk 421 during flaring activity in March 2010, A\&A 578 (2015) A22

[4] S. Aleksić , L. A. Ansoldi, P. Antonelli et al., The 2009 multiwavelength campaign on Mrk 421: Variability and correlation studies, A\&A 576 (2015) A126

[5] A. Furniss , K. Noda, J. Boggs et al., First NuSTAR Observations of Mrk 501 within a Radio to TeV Multi-Instrument Campaign, ApJ 812 (2015) 65

[6] H. Anderhub , M. Backes, A. Biland et al., Design and operation of FACT - the first G-APD Cherenkov telescope, JInst 8 (2013) P06008

[7] D. Dorner et al. (FACT Collaboration), FACT - TeV Flare Alerts Triggering Multi-Wavelength Observations, in proceedings of the 34th International Cosmic Ray Conference (ICRC2015), POS ( ICRC2015) 704 (2015)

[8] D. Dorner et al. (FACT Collaboration), FACT - Highlights from more than Five Years of Unbiased Monitoring at TeV Energies, in proceedings of the 36th International Cosmic Ray Conference (ICRC2017), POS ( ICRC2017) 609 (2017)

[9] A. Biland, T. Bretz, J. Buß et al. Calibration and performance of the photon sensor response of FACT - the first G-APD Cherenkov telescope, JInst 9 (2014) P10012 
[10] A. Biland et al. (FACT Collaboration), FACT: Towards Robotic Operation of an Imaging Air Cherenkov Telescope, in proceedings of the 33th International Cosmic Ray Conference (ICRC2013), arXiv:1307.7892 (2013)

[11] D. Dorner et al. (FACT and HAWC Collaborations) First Study of Combined Blazar Light Curves with FACT and HAWC, in proceedings of 6th International Symposium on Gamma-Ray Astronomy, AIP arXiv:1610.06627 (2016)

[12] D. Dorner, R. Lauer et al. (FACT and HAWC Collaborations) Joint analysis of TeV blazar light curves with FACT and HAWC, in proceedings of the 36th International Cosmic Ray Conference (ICRC2017), POS ( ICRC2017) 625 (2017)

[13] A. Biland et al. (FACT Collaboration), FACT - Status and Experience from Three Years Operation of the First SiPM Camera, in proceedings of the 34th International Cosmic Ray Conference (ICRC2015), POS ( ICRC2015) 1032 (2015)

[14] R. Lauer (HAWC Collaboration), Monitoring the Variable Gamma-Ray Sky with HAWC, AIP arXiv:1610.05172 (2016)

[15] R. Lauer and P. Younk (HAWC Collaboration), Results from monitoring TeV blazars with HAWC, in proceedings of the 34th International Cosmic Ray Conference (ICRC2015), PoS ( ICRC2015) 716 (2015)

[16] M. Backes, J. K. Becker, F. Clevermann et al., Long-Term Monitoring of Bright Blazars with a Dedicated Cherenkov Telescope, IJMPD 18 (2009) 1645-1649

[17] U. Abeysekara, A. G. Akhperjanian, A. R. Bazer-Bachi et al., VAMOS: A pathfinder for the HAWC gamma-ray observatory, APh 62 (2015) 125-133

[18] SensL Data sheets, 2015.

[19] C. Jackson, L. Wall, K. O’Neill et al., Through silicon via developments for silicon photomultiplier sensors, Proc. SPIE 9359 (2015) 93591A

[20] Hamamamatsu, MPPC Datasheets.

[21] T. Bretz et al., FACT - The G-APD revolution in Cherenkov astronomy, Proceedings of the Nuclear Science Symposium and Medical Imaging Conference (IEEE-NSS/MIC), 2013

[22] M. Schaufel, T. Bretz, J. Schumacher et al., Small-size air-Cherenkov telescopes for ground detection arrays - a possible future extension?, PoS(ICRC2017)786.

[23] G. Tovmassian, G. Hernandez, M. S. Ochoa et al., Astroclimatic Characterization of Vallecitos: A Candidate Site for the Cherenkov Telescope Array at San Pedro Mártir, PASP 128 (2016) 035004 